\title{
The drag force in two-fluid models of gas-solid flows
}

\author{
Yonghao Zhang ${ }^{\mathrm{a}}$ and Jason M. Reese ${ }^{\mathrm{b}}$ \\ a Department of Computational Science and Engineering, Daresbury Laboratory, \\ Daresbury, Warrington, WA4 4AD, UK \\ ${ }^{\mathrm{b}}$ Department of Mechanical Engineering, King's College London, London WC2R 2LS, UK
}

\section{Introduction}

Currently, the two most widespread methods for modelling the particulate phase in numerical simulations of gas-solid flows are discrete particle simulation (see, e.g., Mikami, Kamiya and Horio 1998), and the two-fluid approach, e.g. kinetic theory models (see, e.g., Louge, Mastorakos and Jenkins 1991). In both approaches the gas phase is described by a locallyaveraged Navier-Stokes equation and the two phases are usually coupled by a drag force. Due to the large density difference between the particles and the gas, inter-phase forces other than the drag force are usually neglected, so it plays a significant role in characterising the gassolid flow. Yasuna, Moyer, Elliott and Sinclair (1995) have shown that the solution of their model is sensitive to the drag coefficient. In general, the performance of most current models depends critically on the accuracy of the drag force formulation.

\section{Problems with the drag force formulation}

The drag force experienced by a spherical particle of diameter $d$ moving in an infinite fluid of density $\rho_{1}$ is given by

$$
f_{\text {drag }}=\frac{\pi}{8} C_{D} d^{2} \rho_{1}|\overline{\boldsymbol{V}}-\boldsymbol{u}|(\overline{\boldsymbol{V}}-\boldsymbol{u}),
$$

where $\boldsymbol{u}$ is the velocity of the particle, $\bar{V}$ is the fluid velocity at infinity, and $C_{D}$ is the drag coefficient. If the particle is surrounded by many others, and the local particle volume fraction is $\varepsilon_{2}$, the drag force volume-averaged over a cell containing only a single particle should be given by

$$
\overline{\boldsymbol{f}}_{d r a g}=\beta(\boldsymbol{V}-\boldsymbol{u})=\frac{3}{4} C_{D} \frac{\varepsilon_{2} \rho_{1}}{d}|\overline{\boldsymbol{v}}-\boldsymbol{u}|(\overline{\boldsymbol{v}}-\boldsymbol{u}),
$$

where the volume of the cell, $\delta V_{1}=1 / 6 \pi d^{3} / \varepsilon_{2}$, is the characteristic element of volume of a twophase mixture containing a single particle and associated fluid; $\overline{\boldsymbol{v}}$ is the averaged velocity of 
this fluid over $\delta V_{1}$. However, the particle volume fraction has been proven to have a more complex and subtle influence on the drag force (e.g. Wen and Yu 1966; Di Felice 1994). Generally, the momentum transfer coefficient, $\beta$, can be expressed as

$$
\beta=\frac{3}{4} C_{D} \frac{\varepsilon_{2} \rho_{1}}{d}|\overline{\boldsymbol{v}}-\boldsymbol{u}| f\left(\varepsilon_{2}\right),
$$

and many forms for the correction factor $f\left(\varepsilon_{2}\right)$ have been proposed. For example, Di Felice (1994) gave

$$
f\left(\varepsilon_{2}\right)=\varepsilon_{2}^{-\chi}
$$

where $\chi$ is an empirical coefficient, which depends on the particle Reynolds number $\operatorname{Re}_{p}$ via

$$
\chi=3.7-0.65 \exp \left[-\frac{1}{2}\left(1.5-\log _{10} \operatorname{Re}_{p}\right)^{2}\right] .
$$

In discrete particle simulations, the usual expressions for the momentum transfer coefficient are extended from the work of Ergun and Orning (1949), Ergun (1952) and Wen and Yu (1966), where the influence of solid volume fraction is incorporated:

$$
\begin{array}{ll}
\beta=150 \frac{\varepsilon_{2}^{2} \mu}{\varepsilon_{1} d^{2}}+1.75 \frac{\varepsilon_{2} \rho_{1}}{d}|\overline{\boldsymbol{v}}-\boldsymbol{u}|, & \varepsilon_{1}<0.8, \\
\beta=\frac{3}{4} C_{D} \frac{\varepsilon_{2} \rho_{1}}{d}|\overline{\boldsymbol{v}}-\boldsymbol{u}| \varepsilon_{1}{ }^{-2.65}, & \varepsilon_{1} \geq 0.8,
\end{array}
$$

where $\mu$ is the fluid viscosity and $\varepsilon_{1}$ is the local fluid volume fraction such that $\varepsilon_{1}+\varepsilon_{2}=1$. Despite the inconsistency at $\varepsilon_{1}=0.8$ for equations (6) and (7), numerical simulations using these formulations show good agreement with experimental data from pneumatic conveyors and fluidised beds (Kawaguchi, Tanaka and Tsuji 1998; Mikami, Kamiya and Horio 1998; Hoomans, Huipers, Briels and van Swaaij 1996).

However, the work of Ergun and Wen and Yu has also been widely adopted within many two-fluid models for gas-solid flows, where the particulate and fluid velocities are averaged over the much larger volume $\delta V_{2}$ which contains statistically many particles (see Figure 1). For example, Neri and Gidaspow (2000) and Nieuwland, van Sint Annaland, Kuipers and van Swaaij (1996) use momentum transfer coefficients of a similar form to those given in equations (6) and (7) above, viz.

$$
\beta_{1}=150 \frac{\varepsilon_{2}{ }^{2} \mu}{\varepsilon_{1} d^{2}}+1.75 \frac{\varepsilon_{2} \rho_{1}}{d}|\boldsymbol{V}-\boldsymbol{U}|, \quad \varepsilon_{1}<0.8
$$




$$
\beta_{1}=\frac{3}{4} C_{D} \frac{\varepsilon_{2} \rho_{1}}{d}|\boldsymbol{V}-\boldsymbol{U}|_{\varepsilon_{1}^{-2.65}}, \quad \varepsilon_{1} \geq 0.8
$$

where $\boldsymbol{V}$ and $\boldsymbol{U}$ are the gas and particle velocities, respectively, averaged over the element volume $\delta V_{2}$. If we assume that, at least, $\boldsymbol{V}$ equals $\overline{\boldsymbol{v}}$ for the gas, the only difference between equation pairs (6) and (8), and (7) and (9) is whether an instantaneous or an averaged particulate velocity is used.

The original phenomenological Ergun formula is based on observations on a fixed bed where the particles have no relative motion. If it is to be extended to freely moving particles, not only the particle volume fraction but also the random fluctuational velocity of individual particles should be considered. The work of Wen and Yu also only addressed the effect of voidage on the drag force. If we assume the drag force acting on a particle surrounded by others can be expressed by equations (2) and (3), the averaged drag force in a two-fluid model can be re-derived as follows.

\section{A new expression for the averaged drag force}

Anderson and Jackson's (1967) rigorously-derived two-fluid model for particle-fluid flows required volume-averaging the point equations of motion for the fluid and individual particles. In order to smooth out high frequency fluctuations, the elemental volume chosen for this was $\delta V_{2}$, rather than $\delta V_{1}$ (see Figure 1). The choice of the requisite volume element is discussed in Anderson and Jackson (1967).

For gas-solid flows, especially with high particle concentration, the inertial energy loss (which is proportional to the relative velocity squared) is mainly due to local flow contraction, expansion and change in flow direction, rather than gas phase turbulence: this inertial loss also occurs when the gas flow is laminar. As Niven (2002) pointed out: "in fully turbulent flow through a packed bed, local losses will very likely dominate the overall pressure loss". Consequently, the gas turbulence may not be the dominant mechanism in particle-gas momentum and energy exchange. In any case, $\overline{\boldsymbol{v}}$ is also an averaged gas velocity on the element volume of $\delta V_{1}$. Therefore we assume $\overline{\boldsymbol{v}}=\boldsymbol{V}$ in the derivation below and decompose the velocity of a particle as $\boldsymbol{u}=\boldsymbol{U}+\boldsymbol{u}^{\prime}$, where $\boldsymbol{u}^{\prime}$ is the instantaneous fluctuational velocity of the particle. The distribution of particle fluctuational velocity in the elemental volume $\delta V_{2}$ is assumed to be described by the Maxwellian 


$$
f^{(0)}\left(\boldsymbol{u}^{\prime}\right)=\frac{1}{(2 \pi T)^{3 / 2}} \exp \left[-\frac{\boldsymbol{u}^{\prime 2}}{2 T}\right],
$$

where $T$ is the granular temperature, given by $1 / 3\left\langle\boldsymbol{u}^{\prime 2}\right\rangle$.

The averaged drag force over $\delta V_{2}$, containing $n$ particles, i.e. $n$ cells each of volume $\delta V_{1}$, can be given by

$$
\boldsymbol{F}_{d r a g}=\sum_{i=1}^{n} \overline{\boldsymbol{f}}_{d r a g, i}=\int_{-\infty}^{\infty} \overline{\boldsymbol{f}}_{d r a g} f\left(\boldsymbol{u}^{\prime}\right) d\left(\boldsymbol{u}^{\prime}\right)
$$

where $f\left(u^{\prime}\right)$ is particle velocity distribution function. Substituting equations (2) and (3) into this equation, and assuming the gradient of the fluid volume fraction is negligible in the elemental volume $\delta V_{2}$, we obtain

$$
\boldsymbol{F}_{d r a g}=\frac{3}{4} C_{D} \frac{\varepsilon_{2} \rho_{1}}{d} f\left(\varepsilon_{2}\right) \int_{-\infty}^{\infty}\left|\boldsymbol{V}-\boldsymbol{U}-\boldsymbol{u}^{\prime}\right|\left(\boldsymbol{V}-\boldsymbol{U}-\boldsymbol{u}^{\prime}\right) f\left(\boldsymbol{u}^{\prime}\right) d\left(\boldsymbol{u}^{\prime}\right)
$$

where the drag coefficient, $C_{D}$, is treated as an "averaged value" over $\delta V_{2}$. Under the assumed Maxwellian distribution of the particle fluctuation velocity, we find

$$
\int_{-\infty}^{\infty}\left|\boldsymbol{u}^{\prime}\right| f^{(0)}\left(\boldsymbol{u}^{\prime}\right) d \boldsymbol{u}^{\prime}=\sqrt{\frac{8 T}{\pi}} .
$$

If $\sqrt{8 T / \pi}<|\boldsymbol{U}-\boldsymbol{V}|$, which is satisfied by most gas-solid flows in pneumatic conveying systems and circulating fluidised beds, equation (12) then becomes

$$
\boldsymbol{F}_{d r a g} \approx \frac{3}{4} C_{D} \frac{\varepsilon_{2} \rho_{1}}{d}(\boldsymbol{V}-\boldsymbol{U}) f\left(\varepsilon_{2}\right) \int_{-\infty}^{\infty}\left|\boldsymbol{V}-\boldsymbol{U}-\boldsymbol{u}^{\prime}\right| f^{(0)}\left(\boldsymbol{u}^{\prime}\right) d\left(\boldsymbol{u}^{\prime}\right)
$$

If $\boldsymbol{F}_{d r a g}$ is expressed in the standard form $\beta_{0}(\boldsymbol{V}-\boldsymbol{U})$, then,

$$
\begin{aligned}
\beta_{0} & =\frac{3}{4} C_{D} \frac{\varepsilon_{2} \rho_{1}}{d} f\left(\varepsilon_{2}\right) \int_{-\infty}^{\infty}\left|\boldsymbol{V}-\boldsymbol{U}-\boldsymbol{u}^{\prime}\right| f^{(0)}\left(\boldsymbol{u}^{\prime}\right) d\left(\boldsymbol{u}^{\prime}\right) \\
& \approx \frac{3}{4} C_{D} \frac{\varepsilon_{2} \rho_{1}}{d}\left[(\boldsymbol{V}-\boldsymbol{U})^{2}+\frac{8 T}{\pi}\right]^{1 / 2} f\left(\varepsilon_{2}\right) \\
& =\frac{3}{4} C_{D} \frac{\varepsilon_{2} \rho_{1}}{d}\left|U_{r}\right| f\left(\varepsilon_{2}\right) .
\end{aligned}
$$

Here, $U_{r}$ is defined as the mean magnitude of the slip velocity:

$$
U_{r}=\left[(\boldsymbol{V}-\boldsymbol{U})^{2}+\frac{8 T}{\pi}\right]^{1 / 2} .
$$


Because there are many different formulas for the standard $C_{D}$, and further uncertainty is inevitably introduced when considering the turbulence effects etc. on this coefficient, in the derivation of equation (12) $C_{D}$ is treated as a function of $U_{r}$, and de-coupled from the integral procedure. Then, the commonly-adopted expression for the drag coefficient is that for a single particle, given experimentally by Kürten, Raasch and Rumpf (1966),

$$
C_{D}=\left(0.28+\frac{6}{\sqrt{\operatorname{Re}_{p}}}+\frac{21}{\operatorname{Re}_{p}}\right),
$$

which is valid for particle Reynolds numbers between 0.1 and 4000 . The $C_{D}$ used in equation (15) could be extended from equation (17) by using a particle Reynolds number based on the new $U_{r}$, i.e.

$$
\operatorname{Re}_{p}=\frac{\rho_{1} U_{r} d}{\mu}
$$

If the form of $f\left(\varepsilon_{2}\right)$ is that given in equations (6) and (7), the corresponding new expressions for the averaged drag force in a two-fluid model are

$$
\begin{array}{ll}
\beta_{0}=150 \frac{\varepsilon_{2}^{2} \mu}{\varepsilon_{1} d^{2}}+1.75 \frac{\varepsilon_{2} \rho_{1}}{d} U_{r}, & \varepsilon_{1}<0.8, \\
\beta_{0}=\frac{3}{4} C_{D} \frac{\varepsilon_{2} \rho_{1}}{d} U_{r} \varepsilon_{1}^{-2.65}, & \varepsilon_{1} \geq 0.8 .
\end{array}
$$

Both $\beta_{1}$ of equations (8) and (9), and $\beta_{0}$ of equations (19) and (20) incorporate the influence of solid volume fraction. Additionally, $\beta_{0}$ addresses the influence of the relative random motion of the particles.

\section{Discussion}

The impact of this new $\beta_{0}$ on predicted mean slip velocities and pressure drops in a gas-solid system is now discussed; the vertical flow of solid particles in a pipe is a common test-case for two-phase flow models. Predicted particulate axial velocity profiles using the previous expression for the inter-phase momentum transfer coefficient, $\beta_{1}$, as given in equation (9), and the present one, $\beta_{0}$, as given in equation (20), are shown in Figure 2. The experimental data and system parameters are taken from the work of Maeda, Hishida and Furutani (1980). Details of the numerical simulation are given in Zhang and Reese (2001), and the influence of the particle volume fraction on the drag force is treated in the same way as Wen and $\mathrm{Yu}$ (1966). As $\beta_{0}$ takes into account the relative fluctuational motion between the two phases, a 
smaller slip velocity is predicted. This then leads to a lower axial pressure drop along the pipe, which can be seen in Figure 3. There is a negligible difference between profiles of normalised gas axial velocity calculated using $\beta_{0}$ and $\beta_{1}$.

Figure 3 compares the experimental data of Miller and Gidaspow (1992) with the simulation results of the model of Neri and Gidaspow (2000) using $\beta_{1}$, and the present model using $\beta_{0}$. In the two simulations, all other model parameters apart from the momentum transfer coefficient are identical. The physical system examined is, again, vertical pipe flow, with a superficial gas velocity of $2.89 \mathrm{~ms}^{-1}$. The most evident impact of using the new $\beta_{0}$ is that the predicted axial pressure gradient becomes smaller. Simulation results using $\beta_{0}$ show better quantitative agreement with the experimental measurements in both Figures 2 and 3. This indicates that the validity and applicability of the new expression for $\beta_{0}$ for the inter-phase momentum transfer coefficient are worth further exploration.

\section{Conclusions}

In two-fluid models for gas-solid flows, the averaged drag force plays an essential role in coupling the gas and particles. As this drag force has a considerable effect on predicted flow characteristics, it is important to use the most accurate available expressions. The averaged drag force needs not only to incorporate the influence of solid volume fraction but also to address the effect of the random fluctuational motion of the particles.

\section{References}

Anderson, T.B. \& Jackson, R. (1967). Fluid mechanical description of fluidized beds: comparison with theory and experiment. I\&EC Fund. 6, 527.

Di Felice, R. (1994). The voidage function for fluid-particle interaction systems. Int. J. Multiphase Flow 20, 153.

Ergun, S. (1952). Fluid flow through packed columns. Chem. Eng. Prog. 48, 89.

Ergun, S. \& Orning, A.A. (1949). Fluid flow through randomly packed columns and fluidised beds. Ind. Eng. Chem. 41(6), 1179.

Hoomans, B.P.B., Huipers, J.A.M., Briels, W.J. \& Van Swaaij, W.P.M. (1996). Discrete particle simulation of bubble and slug formation in a two-dimensional gas-fluidised bed: a hard-sphere approach. Chem. Eng. Sci. 51, 99. 
Kawaguchi, T., Tanaka, T. \& Tsuji, Y. (1998). Numerical simulation of two-dimensional fluidized beds using the discrete element method (comparison between the two- and threedimensional models). Powder Tech. 96, 129.

Kürten, H., Raasch, J. \& Rumpf, H. (1966) Chem. Ing. Tech. 38, 941.

Louge, M.Y., Mastorakos, E. \& Jenkins, J.K. (1991). The role of particle collisions in pneumatic transport. J. Fluid Mech. 231, 345.

Maeda, M., Hishida, K. \& Furutani, T. (1980). Optical measurements of local gas and particle velocity in an upward flowing dilute gas-solid suspension. Polyphase Flow and Transport Technology, 211. Century 2-ETC, San Francisco.

Mikami, T., Kamiya, H. \& Horio, M. (1998). Numerical simulation of cohesive powder behavior in a fluidized bed. Chem. Eng. Sci. 53, 1927.

Miller, A. \& Gidaspow, D. (1992). Dense, vertical gas-solid flow in a pipe. AIChE J. 38, 1801.

Neri, A. \& Gidaspow, D. (2000). Riser hydrodynamics: simulation using kinetic theory. AIChE J. 46, 52.

Nieuwland, J.J., van Sint Annaland, M., Kuipers, J.A.M., van Swaaij, W.P.M. (1996) Hydrodynamic modelling of gas/particle flows in riser reactors. AIChE J 42, 1569.

Niven, R.K. (2002) Physical insight into the Ergun and Wen \& Yu equations for fluid flow in packed and fluidised beds. Chem. Eng. Sci. 57, 527.

Wen, C.Y. \& Yu, Y.H. (1966). A generalized method for predicting the minimum fluidization velocity. AIChE J. 12, 610 .

Yasuna, J.A., Moyer, H. R., Elliott, S. \& Sinclair, J.L. (1995). Quantitative predictions of gas-particle flow in a vertical pipe with particle-particle interactions. Powder Technol. 84, 23.

Zhang, Y. \& Reese, J.M. (2001). Particle-gas turbulence interactions in a kinetic theory approach to granular flows. Int. J. Multiphase Flow 27, 1945. 


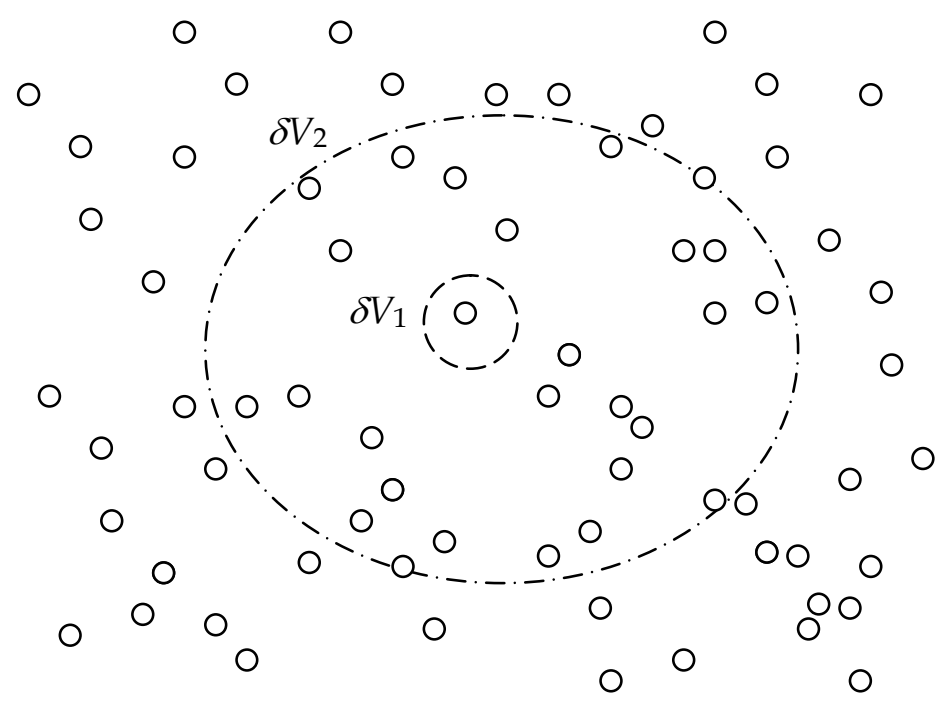

Figure 1 Schematic of the elemental volumes $\delta V_{1}$ and $\delta V_{2}$ in a freely-moving gas-solid flow; solid circles represent particles. The inner broken line represents the boundary of the characteristic volume element $\delta V_{1}$, containing a single particle with local voidage $\varepsilon_{1}$. The outer broken line represents the boundary of $\delta V_{2}$, the elemental volume which contains statistically many freely-moving particles.

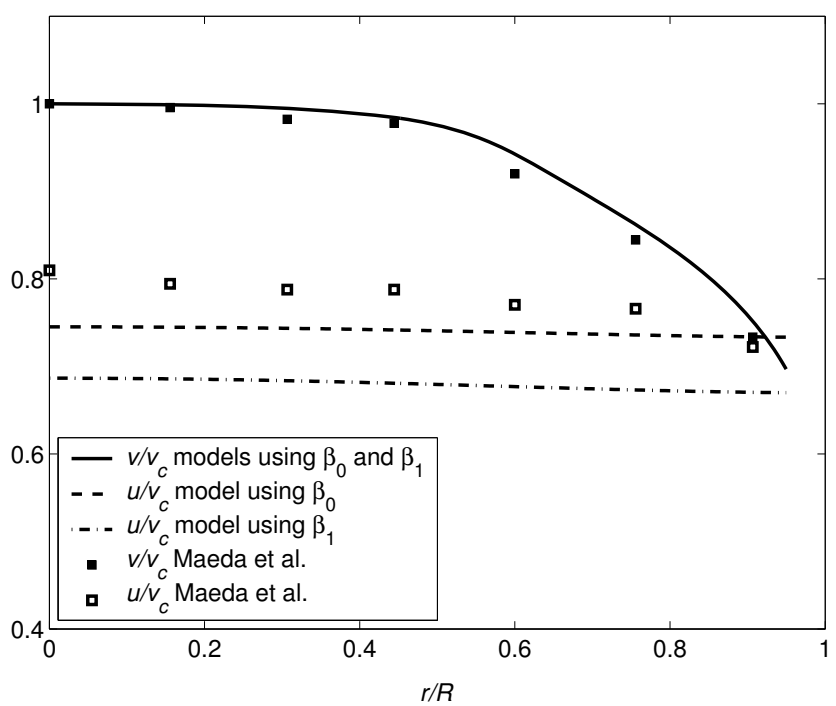

Figure 2 Comparison between numerical predictions (using $\beta_{0}$ and $\beta_{1}$ ) and experimental data for the radial variations of normalized axial velocity of both phases, $v / v_{c}$ and $u / v_{c}$. The mass loading ratio is 0.3 , the Reynolds number 22,000 and the particles are $136 \mu \mathrm{m}$ diameter. Other parameters as in Maeda et al. (1980), which reports the experimental measurements. 


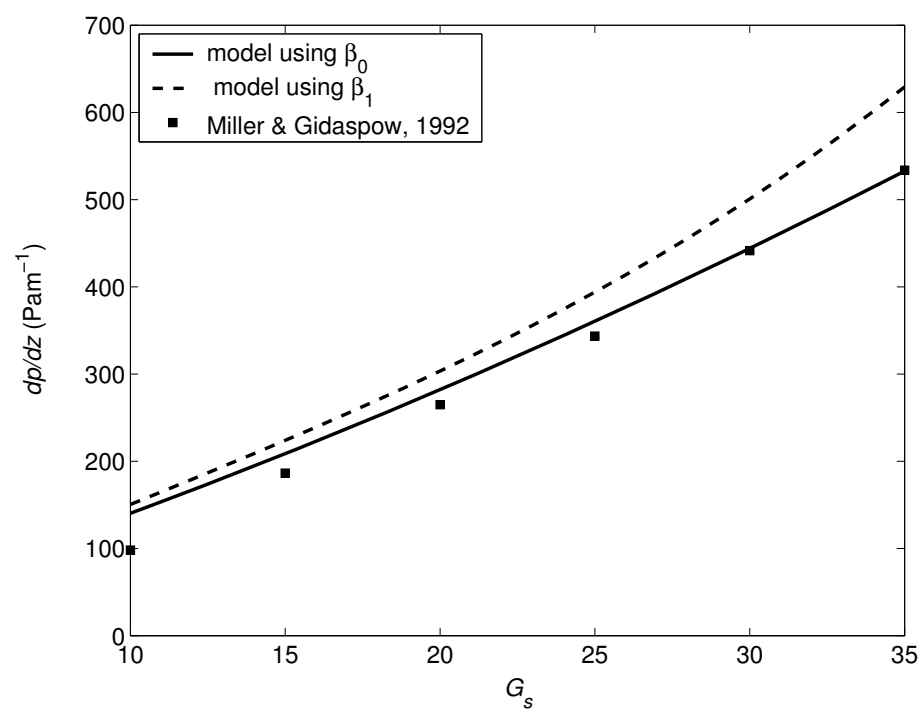

Figure 3 Comparison between numerical predictions (using $\beta_{0}$ and $\beta_{1}$ ) and experimental data for the variation of axial pressure gradient with solid mass flow rate. The superficial gas velocity is $2.89 \mathrm{~ms}^{-1}$ and the particles are $75 \mu \mathrm{m}$ diameter. Other parameters as in Miller and Gidaspow (1992), which reports the experimental measurements. 